\title{
LXXIV. On thialdine and selenaldine, two new artificial organic bases
}

\section{Wöhler \& Liebig}

To cite this article: Wöhler \& Liebig (1847) LXXIV. On thialdine and selenaldine, two new artificial organic bases, Philosophical Magazine Series 3, 30:204, 470-478, DOI: 10.1080/14786444708645761

To link to this article: http://dx.doi.org/10.1080/14786444708645761

册 Published online: 30 Apr 2009.

Submit your article to this journal $[\pi$

Џ Article views: 2

Q View related articles $₫$ 
planes yields common light by reflexion. A truncated hollow cone, the smaller circular section of which was about fourteen lines in diameter, and the larger seventeen lines, was ground into a glass plate, four lines in thickness and three inches in diameter. The lowermost surfaces of the glass were roughened at the margin of the circular section and blackened, and the larger circular section was covered with a glass plate, upon which a circular piece of tin-foil was pasted, so that its centre coincided with the axis of the truncated cone. The angle of the cone at the apex amounted to $70^{\circ} 50^{\prime}$. When this apparatus is turned towards the sun, so that the rays fall at right angles to the base of the cone, they enter the annular space between the tin-foil and the dark coating of the sheath, and arrive at the reflecting surface of the hollow cone at an angle of $35^{\circ} 25^{\prime}$, thus becoming polarized in all the planes of reflexion, and after reflexion, crossing at one point of the axis. In the apparatus made by CErtling, this point was exactly in the plane of the smaller aperture of the cone, and was visible when received upon the surface of a piece of white paper placed there. As the polarized light would be depolarized on account of the depolarizing action of this rough surface, it could only be determined that it was unpolarized. This was effected by polarizing the light incident upon the cone by means of an inserted glass plate, and introducing a plate of mica between the inserted glass plate and the cone. The point remained colourless.

LXXIV. On Thialdine and Selenaldine, two new artificial organic bases. By Wöhler and Liebig. Communicated by Dr. A. W. HoFmanN *.

\section{Thialdine.}

THE peculiar deportment of hydrated cyanic acid with aldehyde, which gives rise to a new acid containing the elements of aldehydite of ammonia and hydrated cyanic acid, induced us to study the action of some other substances on aldehyde and aldehydite of ammonia.

The most interesting product which we obtained along with others, is a new organic alkali free from oxygen, and containing carbon and hydrogen in the same proportions as acetic acid, combined with sulphur and the elements of sulphide of ammonium. This substance, which we call thialdine $\phi$, is highly remarkable from its composition, and we have no doubt that the method which led to its discovery, when applied to other compounds analogous to aldehyde and aldehydite of ammonia, will yield a long series of new and inter-

* Communicated by the Chemical Society; having been read January 4, 1847 . $\uparrow$ Contracted from $\theta \in i o \nu$ and aldehyde. 
esting products. The study of these substances promises to become highly important for the theory of the formation of organic bases, in which the action of ammonia is frequently very prominent, and it may be expected that a close investigation of the production of organic substances containing sulphur will tbrow some light upon their generation in the organism of the plant.

The preparation of thialdine is very simple. One part of aldehydite of ammonia free from æether and alcohol is dissolved in from twelve to sixteen parts of water; one ounce of this liquid is mixed with from ten to fifteen drops of solution of ammonia, and treated with a slow stream of hydrosulphuric acid; after half an hour the solution begins to become turbid, and crystals are deposited of the appearance of camphor and of considerable size; after four or five hours the liquid again becomes clear and the operation is terminated. The crystals are collected on a funnel and washed with water until all the sulphide of ammonium is separated. Pressure between bibulous paper removes the adhering water. The dry crystals are dissolved in æther, the "solution mixed with a third of its volume of alcohol and left to spontaneous evaporation. The liquid soon deposits very regular rhombic tables, which can be obtained of half an inch in dimensions if too rapid an evaporation of the solution has been avoided.

If no more of the solution is left than is required to cover the crystals formed, the mother-liquid is decanted off; the crystals are then dried by leaving them for some time between folds of bibulous paper. The mother-liquor yields some more thialdine on evaporation. The crystals thus obtained are however not quite colourless. The remaining liquid contains much sulphide of ammonium, part of which arises from the ammonia added.

It happens sometimes that on passing hydrosulphuric acid gas through the solution of aldehydite of ammonia, no crystalline body, but a heavy colourless oil of offensive odour is deposited. This oil is a mixture of two bodies, the greater part being thialdine, the fusing-point of which is depressed to the temperature of the atmosphere by the presence of a liquid substance*. In order to obtain pure thialdine from this mixture, the supernatant aqueous layer is separated, and the remaining liquid (consisting principally of the oil and but a small amount of the aqueous solution) is agitated with half its bulk of rther, which instantaneously dissolves the oil. The æethereal solution, which may be easily separated from

* Crystals of thialdine when placed in contact with sulphide of ammonium for some time are entirely converted into a heavy oil, insoluble in water, which has not been further examined. 
the water, is agitated in a stoppered bottle with a little concentrated hydrochloric acid. The mixture generally solidifies as a mass of fine needles, which may be separated from the oil by washing with æether. In this manner hydrochlorate of thialdine is obtained, from which pure thialdine may easily be prepared by moistening the dry crystals with a concentrated solution of ammonia, and extracting the separated base by means of æther. The æthereal solution yields by spontaneous evaporation crystals of pure thialdine. Addition of alcohol to the æethereal solution facilitates the crystallization exceedingly; the crystals become larger, more regular, and perfectly transparent.

Pure thialdine is heavier than water; its specific gravity is $1 \cdot 191$ at $18^{\circ} \mathrm{C}$.; it forms large, transparent, colourless, shining crystals of the form of common gypsum, possessing a highly refracting power and a peculiar aromatic, but after some time very disagreeable odour. The crystals fuse at $43^{\circ} \mathrm{C}$; ; the fused crystals solidify at $42^{\circ}$; they are volatile at the temperature of the atmosphere without residue. Thialdine distils with the vapour of water, but is decomposed when distilled alone, a heavy oil of offensive odour passes over, solidifying only partly and after some time, whilst a brown, syrupy residue containing sulphur is left in the retort. Thialdine in this respect resembles aldehydite of ammonia, which, though volatile itself, cannot be exposed to a higher temperature without decomposition. If a crystal of thialdine remains for several hours in an atmosphere containing acid vapours, such as is the case in the laboratory, a white envelope of fine silky needles is found at a certain distance from the planes of the crystals.

Thialdine is but little soluble in water; it is soluble in alcohol, and much more so in ather; when powdered it deliquesces at the common temperature in vapour of æether, or in an atmosphere containing vapour of æther.

An alcoholic solution of thialdine exhibits the following reactions :-

With acetate of lead, at first no precipitate, but after some time a yellow deposit appears, which soon becomes red and finally black.

Nitrate of silver causes a precipitate, which at first is white, then yellow, afterwards black.

With chloride of mercury a white precipitate is produced, turning rapidly yellow.

With bichloride of platinum a dingy, yellow precipitate is thrown down after some time.

Thialdine has no reaction on vegetable colours; it is soluble in all acids with which it combines, forming crystallizable salts; the hydrochlorate and the nitrate are remarkable for 
the facility with which they crystallize and for the beauty of the crystals.

Thialdine and its salts are decomposed when gently heated with a solution of nitrate of silver; sulphide of silver is produced with disengagement of a combustible gas, easily inflammable and possessing the odour and all the properties of aldehyde; namely, it is entirely absorbed by concentrated sulphuric acid and solution of potassa, the former becoming brown immediately, the latter remaining clear but depositing when gently heated a yellowish-brown precipitate of resin of aldehyde, and assuming the peculiar and offensive odour which accompanies the decomposition of aldehyde under these circumstances.

When thialdine is ignited with lime, chinoline appears among the products of decomposition, which may be easily recognised by its characteristic odour and by its beautiful platinum salt.

With cyanide of mercury thialdine exhibits a peculiar comportment. On mixing the two solutions a white precipitate is produced, which on boiling is converted into amorphous, black sulphide of mercury. If this decomposition takes place in a retort its neck becomes coated with fine crystalline needles, extremely volatile, insoluble in water but easily soluble in alcohol and ether. This new body, in which part or all the sulphur appears to be replaced by cyanogen, is formed in but small quantities, which up to this moment has prevented us from studying it any further.

The analysis of thialdine was performed in the usual way; the combustion with oxide of copper is attended with difficulties, as the conversion of carbon into carbonic acid is obstructed by the large quantity of sulphur contained in the body forming a corresponding amount of sulphide of copper at the point of contact between the substance and oxide of copper.

On burning thialdine with oxide of copper and separating the sulphurous acid formed by means of peroxide of lead, a gas is obtained containing volumes of nitrogen and carbonic acid in the proportion of $1: 12$. Aldehydite of ammonia, from which thialdine is produced, contains 4 equivs. of carbon to 1 equiv. of nitrogen, from which it is evident that aldehydite of ammonia on being converted into thialdine by means of hydrosulphuric acid has lost two-thirds of its nitrogen.

We have mentioned that thialdine, on being gently heated with a solution of nitrate of silver, has its sulphur converted into sulphide of silver. This mode of decomposition was employed to determine the sulphur. The nitrogen of the thialdine remains in the residuary liquid in the form of ammonia, from 
which it was precipitated by bichloride of platinum, and determined in the usual way*.

$0.3633 \mathrm{grm}$. of thialdine gave $0.5845 \mathrm{grm}$. of carbonic acid and $0.267 \mathrm{grm}$. of water.

$0.509 \mathrm{grm}$. of thialdine gave $0.816 \mathrm{grm}$. of carbonic acid and 0.3720 of water.

$0.4508 \mathrm{grm}$. of thialdine gave $0.566 \mathrm{grm}$. of ammoniochloride of platinum.

$0.6430 \mathrm{grm}$. of thialdine gave $1.923 \mathrm{grm}$. of sulphide of silver.

$0.3140 \mathrm{grm}$. of thialdine gave $0.8420 \mathrm{grm}$. of water.

The atomic weight having been ascertained by the analysis of the hydrochlorate and nitrate, the preceding numbers lead to the following formula-

$$
\mathrm{C}_{12} \mathrm{H}_{13} \mathrm{~N} \mathrm{~S}_{4} \text {, }
$$

as will be seen on comparing the theoretical and experimental numbers.

\begin{tabular}{|c|c|c|c|c|c|}
\hline \multirow{2}{*}{\multicolumn{3}{|c|}{12 eqs, of Carbon }} & \multirow{2}{*}{\multicolumn{2}{|c|}{$\begin{array}{r}\text { Theory. } \\
44 \cdot 17\end{array}$}} & experiments. \\
\hline & & & & & 43.80 \\
\hline 13 & $\ldots$ & Hydrogen & 13 & $8 \cdot 04$ & $8 \cdot 04$ \\
\hline 1 & ... & Nitrogen . & 14 & $8 \cdot 58$ & $8 \cdot 54 \uparrow$ \\
\hline 4 & $\ldots$ & Sulphur . & - $\frac{64}{163}$ & $39 \cdot 26$ & $39 \cdot 14$ \\
\hline
\end{tabular}

Hydrochlorate of Thialdine.-Thialdine is dissolved in large quantity by hydrochloric acid: the saturated solution has an acid reaction. On spontaneous evaporation, or concentration on the water-bath, it deposits large, regular, transparent prisms of great lustre, and frequently an inch in length. The hydrochloric solution of crude thialdine contains in admixture a foreign body of most offensive odour; on agitating with æther this odour disappears immediately, and the liquid which formerly was turbid becomes perfectly transparent. Hydrochlorate of thialdine is rather soluble in water, less so in alcohol; both liquids dissolve a large quantity when hot, and deposit beautiful and perfectly regular crystals on cooling. Hydrochlorate of thialdine is insoluble in æether. When heated in the dry state this salt is decomposed without fusion; it becomes brown, and chloride of ammonium sublimes with evolution of a gas possessing a most offensive odour and burning with a dull flame. From the solution of this salt nitrate of silver throws down a yellow precipitate, which, on being gently heated, turns black with evolution of aldehyde. This black precipitate is a mixture of chloride and sulphide of silver; all the nitrogen of the base remains in the liquid in the form of ammonia.

* The following analyses were made by Dr. Strecker at Giessen, and Dr. Städler in Göttingen.

+ Calculated from the proportion of $12 \mathrm{C}: 1 \mathrm{~N}$. 
$0.4577 \mathrm{grm}$. of hydrochlorate of thialdine gave $0.5933 \mathrm{grm}$. of carbonic acid and $0.2850 \mathrm{grm}$. of water, corresponding to 35.35 per cent. of carbon and 6.92 per cent. of hydrogen.

$0.726 \mathrm{grm}$. of hydrochlorate of thialdine gave $0.789 \mathrm{grm}$. of ammonio-chloride of platinum.

$0.7735 \mathrm{grm}$. of hydrochlorate of thialdine gave $0.8225 \mathrm{grm}$. of ammonio-chloride of platinum. In the mean, 6.79 per cent. of nitrogen.

$0.7798 \mathrm{grm}$. of hydrochlorate of thialdine gave $1.890 \mathrm{grm}$. of sulphide of silver $=32.09$ per cent. of sulphur.

$0 \cdot 7735 \mathrm{grm}$. of hydrochlorate of thialdine gave $1.915 \mathrm{grm}$. of sulphide of silver $=31.92$ per cent. of sulphur.

$0.7598 \mathrm{grm}$. of hydrochlorate of thialdine was precipitated with nitrate of silver and boiled until evolution of gas had ceased; the precipitate, when extracted with ammonia and precipitated with nitric acid, gave $0.5283 \mathrm{grm}$. of chloride of silver $=17 \cdot 14$ per cent. of chlorine.

$0.7735 \mathrm{grm}$. treated in the same way, gave $0.5405 \mathrm{grm}$. of chloride of silver $=17 \cdot 24$ per cent. of chlorine.

$0.776 \mathrm{grm}$. dissolved in alcohol and precipitated in the same manner with nitrate of silver, gave $1.912 \mathrm{grm}$. of sulphide of silver $=31.903$ per cent. of sulphur and 0.551 grm. of chloride of silver $=17.551$ per cent. of chlorine.

$0.441 \mathrm{grm}$. ignited with a mixture of lime and nitrate of potassa, gave $0.32 \mathrm{grm}$. of chloride of silver $=17.94$ per cent. of chlorine.

These results lead to the following formula for hydrochlorate of thialdine,-

$$
\mathrm{C}_{12} \mathrm{H}_{13} \mathrm{NS}_{4}, \mathrm{HCl}
$$

as may be seen from the following comparison between the theoretical and experimental numbers :-

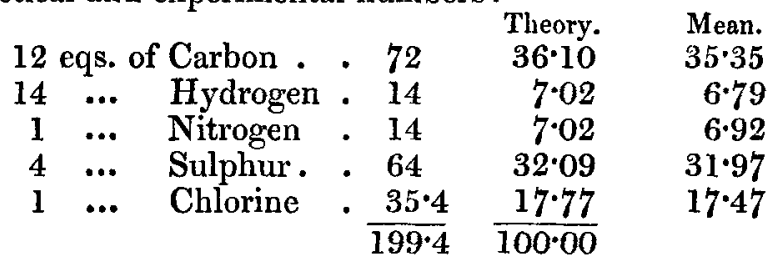

Nitrate of Thialdine.-This salt may be obtained by mixing this base directly with dilute nitric acid; it is, however, preferable to dissolve crude thialdine in æather, and agitate the liquid with moderately concentrated nitric acid; the solution solidifies into a crystalline mass, which is washed with xther, dissolved in water, and crystallized by evaporation and cooling.

Nitrate of thialdine forms fine white needles, more soluble in water than the hydrochlorate; they are soluble in cold, and more so in hot alcohol, from which liquid they likewise 
recrystallize on cooling: they are insoluble in æther; on heating they fuse and are decomposed.

On burning this salt with chromate of lead, more accurate numbers for the carbon and hydrogen were obtained than in the analysis of thialdine itself or the hydrochlorate, a result which can easily be explained by the co-operation of the oxygen in the nitric acid.

$0.357 \mathrm{grm}$. of nitrate of thialdine gave $0.4155 \mathrm{grm}$. of carbonic acid and 0.2045 grm. of water.

$0.514 \mathrm{grm}$. of nitrate of thialdine gave $1.116 \mathrm{grm}$. of sulphide of silver.

$0.6696 \mathrm{grm}$. of nitrate of thialdine, when ignited with a mixture of potassa and nitre, gave $1.4063 \mathrm{grm}$. of sulphate of baryta; the mean of the two latter experiments equals $28^{\circ} 4$ per cent. of sulphur.

These results lead to the following formula for nitrate of thialdine, - $\quad \mathrm{C}_{12} \mathrm{H}_{13} \mathrm{~N} \mathrm{~S}_{4}, \mathrm{H} \mathrm{NO}_{6}$,

as will be seen by the following comparison between the theoretical and experimental numbers:-

$$
\begin{array}{rlll}
12 & \text { eqs. } & \text { of Carbon } & 72 \\
14 & \ldots & \text { Hydrogen } & 14 \\
2 & \ldots & \text { Nitrogen } 28 \\
4 & \ldots & \text { Sulphur } & 64 \\
6 & \ldots & \text { Oxygen } & \frac{.48}{226}
\end{array}
$$

$\begin{array}{cc}\text { Theory. } & \text { Experiment. } \\ 31 \cdot 80 & 31 \cdot 75 \\ 6 \cdot 19 & 6 \cdot 36 \\ 28 \cdot 34 & 28 \cdot 40\end{array}$

The formation of thialdine is easily accounted for : 3 eqs. of aldehydite of ammonia and 6 eqs. of hydrosulphuric acid transpose into 1 eq. of thialdine, 6 eqs. of water, and 2 eqs. of sulphide of ammonium.

$$
\begin{aligned}
& 3 \text { eqs. of Aldehydite of ammonia } ._{12} \mathrm{C}_{21} \mathrm{~N}_{3} \quad \mathrm{O}_{6} \\
& 6 \ldots \text { of Hydrosulphuric acid . } \cdot \frac{\mathrm{H}_{6}}{\mathrm{C}_{12} \mathrm{H}_{27} \mathrm{~N}_{8} \mathrm{~S}_{6} \mathrm{O}_{6}} \\
& 1 \text { eq. of Thialdine . . . . }, \mathrm{C}_{12} \mathrm{H}_{13} \mathrm{~N} \mathrm{~S}_{4} \\
& 6 \text { eqs. of Water . . . . . . }{ }^{\mathrm{H}_{6}} \quad \mathrm{O}_{6} \\
& 2 \ldots \text { of Sulphide of ammonium } \cdot \frac{\mathrm{H}_{8} \mathrm{~N}_{2} \mathrm{~S}_{2}}{\mathrm{C}_{12} \mathrm{H}_{27} \mathrm{~N}_{3} \mathrm{~S}_{6} \mathrm{O}_{6}}
\end{aligned}
$$

But the question respecting the real constitution of this body is too closely connected with that of the true constitution of organic bases in general to admit of any definite opinion being promulgated at this moment, when so few experiments on the subject are as yet before us. Still we can imagine how important for the general question facts like the formation of thialdine may hereafter become, and that just from this quarter perhaps a way for its solution may be ob- 
tained. We will here merely allude to an explanation of the constitution and basic character of thialdine, at once obvious, namely, that it may be regarded as a copulated compound of sulphide of ammonium with 3 eqs. of a body

$$
\mathrm{C}_{4} \mathrm{H}_{3} \mathrm{~S} \text {, }
$$

which is the sulphide corresponding to the oxide in aldehyde, namely, sulphide of acetyl.

\section{Selenaldine.}

The existence of thialdine and the mode in which this base is formed, induced us to try the preparation of a corresponding selenium compound. We perfectly succeeded in obtaining that body : selenaldine, however, is so soon and easily altered, that we have not as yet been able to subject it to a more profound investigation.

Selenaldine was produced by passing into a concentrated solution of aldehydite of ammonia hydroselenic acid gas, disengaged from selenide of iron by means of sulphuric acid. In order to prevent the decomposition of hydroselenic acid by the oxygen of the atmosphere, the whole apparatus had been filled previously with hydrogen. The excess of the poisonous hydroselenic acid was condensed in a potassa apparatus. After some time the solution of aldehydite of ammonia begins to become turbid, and crystals of selenaldine are deposited. When the mass of crystals is no longer augmented, the excess of hydroselenic acid is expelled by a stream of hydrogen gas; the solution of selenide of ammonium, covering the crystals, which soon turns red and deposits selenium, is now displaced by introducing a stream of cold water, free from atmospheric air, through one of the glass tubes connected with the bottle. The crystals are then collected upon a filter, pressed between folds of bibulous paper and dried over sulphuric acid.

Selenaldine, as it is deposited from the liquid in which it forms, presents itself in small colourless crystals, doubtless isomorphous with thialdine. In contact with the atmosphere it turns immediately yellow. This substance has a feeble but disagreeable odour; it is slightly soluble in water, on which account it should not be washed too long. The aqueous solution and the last washings become soon turbid in contact with the atmosphere, and deposit an orange-yellow body. The same deportment is exhibited by the solution of selenaldine in alcohol and æether, in both of which it is easily soluble. In consequence of its being so easily altered, we did not succeed in obtaining this base in crystals from any of the solutions mentioned. On evaporating them in vacuo over 
sulphuric acid, the greater portion of the substance is volatilized with decomposition, the yellow body being likewise produced, while sulphate of ammonia is formed in the sulphuric acid. Also, when heated alone, selenaldine is decomposed, with disengagement of a gas of most offensive odour. Selenaldine is a base like thialdine; it is soluble in dilute hydrochloric acid, and reprecipitated by ammonia as a crystalline mass. 'The hydrochloric solution also immediately decomposes with evolution of a most offensive odour. Selenaldine is altered in a similar manner by boiling with water. The yellow body, the formation of which seems to be always attended with a liberation of aldehydite of ammonia, when collected is orange-yellow, amorphous, soluble in alcohol and ather, and fuses, when heated with water, to a reddish yellow mass, which remains soft for a long time. When heated alone, this body is charred with evolution of an oil containing selenium and possessing a most offensive odour.

We did not obtain a decisive result when trying to produce a telluraldine, the tellurium which we employed in the preparation of the hydrotelluric acid accidentally containing so large an amount of selenium, that merely selenaldine was deposited from the purple solution, containing, as it appeared, only telluride of ammonium. We intend to repeat this experiment with tellurium free from selenium.

LXXV. Some Remarks on the Air and Water of Towns. By Robert Angus Smith, Ph.D.*

HAVING given considerable attention to the inquiry into the causes affecting the health of towns, I was anxious to find what the real evil in their polluted atmosphere consisted of; the air has been frequently examined, but the differences found do not sufficiently account for the differences perceptible in breathing for the first time in entering a large town from the country, or for the very great difference in the colour and appearance of both when contrasted side by side, by an individual outside a town, having both before his eyes. The accumulation is great in this case, it is true, but therefore so much the more fitted for showing us its true character.

I first examined the rain-water taken from a cistern; a little was boiled down and the solid residue was like fatty matter, which burnt and gave the smell of a fat, leaving afterwards a strong odour of nitrogenized organic matter. As this amounted to nearly one per cent., I was disposed to con$18+7$.

* Communicated by the Chemical Society ; having been read January 4, 